\title{
Clinical Outcomes and Safety of Apatinib Mesylate in the Treatment of Advanced Non-Squamous Non-Small Cell Lung Cancer in Patients Who Progressed After Standard Therapy and Analysis of the KDR Gene Polymorphism
}

This article was published in the following Dove Press journal: OncoTargets and Therapy

\section{Zi-Zheng Song (1D \\ Li-Fen Zhao (iD ${ }^{2}$ \\ Jing Zuo ${ }^{3}$ \\ Zhi-Song Fan ${ }^{3}$ \\ Long Wang ${ }^{3}$ \\ Yu-Dong Wang ${ }^{3}$}

'Department of Medical Oncology, The Affiliated Hospital of Hebei University, Baoding, People's Republic of China; ${ }^{2}$ Department of Respiratory and Critical Care Medicine, The Shanxi Dayi Hospital, Taiyuan, People's Republic of China;

${ }^{3}$ Department of Medical Oncology, The Fourth Hospital of Hebei Medical

University, Shijiazhuang, People's Republic of China
Correspondence: Yu-Dong Wang Department of Medical Oncology, The Fourth Hospital of Hebei Medical University, Shijiazhuang, Hebei 0500II, People's Republic of China Tel +86 I59 31116 6600 Emailwyd_999@I26.com
Purpose: This study investigated the clinical outcomes and safety of apatinib mesylate in the treatment of advanced non-squamous non-small cell lung cancer (NSCLC) in patients who progressed after standard therapy, and analyzed the kinase insert domain receptor $(K D R)$ gene polymorphism.

Methods: A total of 135 patients with advanced non-squamous NSCLC who received apatinib mesylate were included. Objective response rates were evaluated. Subsequently, progression-free survival (PFS) and overall survival (OS) were assessed and safety data were recorded. Additionally, peripheral blood and biopsy cancer tissue specimens were collected from the patients with NSCLC for the genotyping of the genetic polymorphism and mRNA expression of the KDR gene, respectively. Analysis on the association between genotypes and prognosis was conducted. Results: The objective response rate of the 135 patients with NSCLC was $18.52 \%$, disease control rate was $65.19 \%$, median PFS was 3.95 months, and median OS was 10.05 months Regarding the $K D R$ gene polymorphism analysis, the distribution of the 4397T $>$ C polymorphism genotypes was in accordance with the Hardy-Weinberg Equilibrium $(P=0.868)$. Moreover, the prognosis analysis indicated that the median PFS of patients with the CC/TC and TT genotypes was 2.80 and 4.80 months, respectively $(P=0.002)$. Furthermore, the median OS of patients with the two genotypes was 9.10 and 10.56 months, respectively $(P=0.041)$. The multivariate Cox regression analysis showed that the TC/CC genotypes were an independent factor for PFS (odds ratio: $1.72, P=0.009$ ). There was no correlation between the polymorphism and adverse reactions. Additionally, the mRNA expression analysis suggested that the mRNA levels of $K D R$ in cancer tissues were significantly different between the TT and TC/CC genotypes $(P<0.001)$.

Conclusion: The clinical outcomes of treatment with apatinib mesylate for advanced nonsquamous NSCLC in patients who progressed after standard therapy may be influenced by the KDR 4397T $>$ C polymorphism through mediation of the mRNA expression of KDR.

Keywords: non-small cell lung cancer, apatinib mesylate, kinase insert domain receptor, polymorphism, clinical outcomes, safety

\section{Introduction}

Lung cancer is the most common malignancy and leading cause of cancer-related death worldwide. In 2018, it accounted for approximately 2.1 million new cases and 1.77 million deaths globally. ${ }^{1}$ Annually, there are approximately 0.733 million new 
cases and 0.61 million lung cancer-related deaths in China. $^{2}$ In recent years, great progress has been achieved in the treatment of non-small cell lung cancer (NSCLC) in terms of molecular and targeted therapy. This advancement has rendered advanced NSCLC the most successful type of cancer in precision medicine. ${ }^{3}$ However, approximately $50 \%$ of patients with NSCLC in China do not carry a clinically significant driver gene mutation and could only receive treatment with a platinum-containing double-drug chemotherapy regimen as first-line therapy. ${ }^{4}$ According to the Eastern Cooperative Oncology Group (ECOG) 4599 and BEYOND study, bevacizumab combined with chemotherapy was effective in patients with limited survival benefits. ${ }^{5,6}$ The advantages of traditional second-line therapy were expected, and the immunotherapy drugs had been recently approved in mainland China with high cost and relatively limited use among patients. Therefore, patients with NSCLC are in urgent need of effective therapeutic drugs after standard treatment. Encouragingly, an anti-angiogenic small molecule tyrosine kinase inhibitor, termed anlotinib hydrochloride, has become a new standard therapy for third-line treatment in China. ${ }^{7}$ However, anlotinib was licensed by the China State Food and Drug Administration (cFDA) in 2018, and its clinical use in patients with NSCLC was relatively rare.

Apatinib mesylate was the first small-molecule antiangiogenesis targeted drug with demonstrable efficacy and safety in advanced gastric cancer, ${ }^{8}$ which was approved by the cFDA in October 2014 as subsequent-line treatment for advanced or metastatic gastric cancer. An important therapeutic target of apatinib mesylate was the kinase insert domain receptor $(K D R)$, which inhibits tumor angiogenesis by blocking its downstream signaling pathway. ${ }^{9}$ Additionally, numerous clinical research studies supported the effectiveness of apatinib mesylate in NSCLC, colorectal cancer, and breast cancer. ${ }^{10}$ However, currently, there are great individual differences in its clinical application. To the best of our knowledge, the objective response rate (ORR) in the clinical application of anti-angiogenic drugs was generally low. In advanced NSCLC, the ORR to monotherapy with anlotinib, fruquintinib, and apatinib mesylate was $9.18 \%, 16.4 \%$, and $4 \%$, respectively. ${ }^{11}$ Therefore, the investigation of the sensitive population to vascular targeted drugs has been a research hotspot in the field of anti-angiogenesis therapy. ${ }^{12}$

The KDR gene is located on chromosome $4 \mathrm{q} 12$, which contains 30 exons and exhibits large differences between ethnic groups. The mRNA expression of the $K D R$ gene also differs among different study cohorts. ${ }^{13}$ At present, there are few studies investigating the polymorphism of this gene in the Chinese population. The $-906 \mathrm{~T}>\mathrm{C}$ polymorphism is located in the coding region of the KDR gene. Previous studies showed that this polymorphism was significantly correlated with pathological complete response in patients with advanced breast cancer receiving capecitabine-based neoadjuvant therapy. ${ }^{14}$ In addition, studies involving European and American populations found that this polymorphism was associated with overall survival (OS) in patients with advanced renal cell carcinoma receiving sorafenib; nevertheless, the underlying mechanisms have not been thoroughly investigated. ${ }^{15}$ Therefore, the aim of the present study was to investigate the clinical outcomes and safety of apatinib mesylate in the treatment of advanced non-squamous NSCLC in patients who progressed after standard therapy. Moreover, an analysis of the KDR gene polymorphism was performed. Additionally, the influence of this polymorphism on the mRNA expression of the $K D R$ gene in biopsy tissue specimens was also assessed to gain insight into the detailed mechanism involved in this process.

\section{Materials and Methods}

\section{Study Design and Therapeutic Schedule}

This study was a retrospective analysis of apatinib mesylate in patients with NSCLC in China. Therefore, patients with advanced NSCLC who progressed or relapsed after standard therapy from January 2015 to December 2018 in the Department of Medical Oncology of The Fourth Hospital of Hebei Medical University (Shijiazhuang, China) were included in this study. The eligibility criteria included: 1) administration of apatinib mesylate as third- or subsequentline therapy in patients who had received at least two lines of systemic chemotherapy or could not tolerate the treatment; 2 ) patients with an EGFR mutation, $A L K$ rearrangement, ROS1 rearrangement, or other driven gene mutation who received therapy with the corresponding-targeted drug and progressed, and subsequently received treatment with apatinib mesylate; 3 ) age $\geq 18$ years; 4 ) an ECOG performance status of $0-2$; and 5) at least one measurable target lesion according to the response evaluation criteria in solid tumors (RECIST 1.1). The exclusion criteria were: 1) diagnosis of squamous cell lung carcinoma or small cell lung cancer; 2) recent diagnosis of central nervous system metastases; 3 ) presence of concomitant tumors or serious diseases; 4) hemoptysis $>50 \mathrm{~mL}$ per day; and 5) missing efficacy evaluation or follow-up data. The flow chart of our retrospective study is illustrated in Figure 1. The primary endpoint of this 


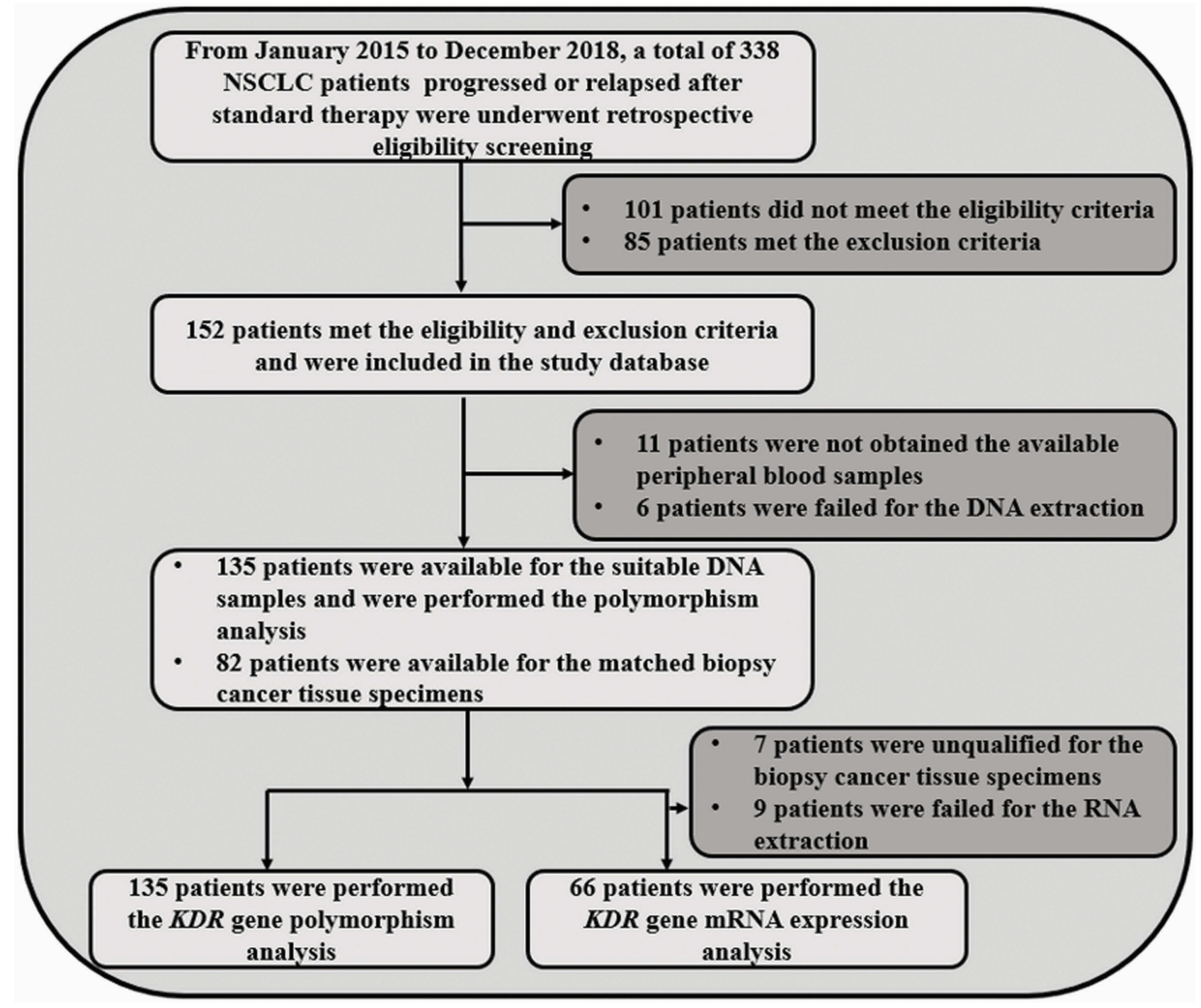

Figure I Flow chart of the retrospective study of apatinib mesylate in the treatment of advanced non-squamous non-small cell lung cancer in patients who progressed after standard therapy.

study was progression-free survival (PFS), while the secondary endpoints were ORR, OS, and the analysis of the $K D R$ gene polymorphism.

Apatinib mesylate was orally administered at an initial dosage of 500-750 mg per day, $30 \mathrm{~min}$ after meals, with warm water, and continuously for 28 days until disease progression or intolerable adverse reactions. The precise dose of apatinib mesylate was determined according to the baseline physical conditions of the patients: body surface area, ECOG score and age. The selection of the dosage range was based on the clinical experience that the recommended dose of $850 \mathrm{mg}$ in the instructions was not tolerable in most patients. The dose of apatinib mesylate was adjusted according to the hematological or non-hematological toxicity during the treatment. The treatment was discontinued following the occurrence of a potentially life-threatening toxic reaction. This study was approved by the ethics committee of The Fourth Hospital of Hebei Medical University. Informed consent was provided by all patients or their family members in accordance with the tenets of the Declaration of Helsinki.

The efficacy was assessed according to the RECIST 1.1 criteria. ${ }^{16}$ Computed tomography was used to assess the change in target lesions after the completion of the first cycle, and subsequently every two cycles, or depended on the actual condition of the patients. The occurrence of adverse reaction during treatment was evaluated using the Common Terminology Criteria for Adverse Events 4.03 version to document hematological and non-hematological events that may be drug-related. ${ }^{17}$ Adverse events with an incidence $\geq 10 \%$ were recorded and analyzed. 


\section{Collection of Peripheral Blood Specimens} and Genotyping of the KDR Gene Polymorphism

Genomic DNA was extracted from whole venous blood (approximately $4 \mathrm{~mL}$ ) collected prior to treatment with apatinib mesylate using the phenol chloroform method according to the standard procedure. The 4397T $>C$ (rs2071559) polymorphism of the $K D R$ gene was genotyped through polymerase chain reaction-restriction fragment length polymorphism (PCR-RFLP). Initially, the PCR product of this polymorphism was amplified. The forward primer was 5'TTCCAAGACCATAGCTTACCA-3' and the reverse primer was 5'-AATGTTTACCAAAGCCCAGA-3'. The size of the PCR product was $297 \mathrm{bp}$. Part of the PCR product $(2 \mu \mathrm{L})$ was digested using the restriction enzyme NdeI (Thermo Fisher Scientific, Waltham, MA, USA). The genotypes of the polymorphism were determined by the size of the PCR bands as follows: CC genotype (one 297-bp band); TT genotype (one 199-bp band and one 98-bp band); TC genotype (one 297-bp band, one 199-bp band, and one 98-bp band).

\section{Collection of Biopsy Cancer Tissue Specimens and Analysis of the KDR Gene mRNA Expression}

Biopsy cancer tissue specimens from 66 randomly selected samples of patients with NSCLC were collected and preserved in liquid nitrogen. Total RNA samples were extracted using the TRIzol reagent (Takara Biotechnology, Dalian, China) according to the instructions provided by the manufacturer and stored at $-80^{\circ} \mathrm{C}$ for mRNA expression analysis. RNA (a total of $500 \mathrm{ng}$ ) extracted from each biopsy cancer tissue specimen was used as the template for reverse transcription-PCR to prepare the first-stand of cDNA using the PrimeScript RT reagent kit (TAKARA Biotechnology, Dalian, China). Relative quantitative analysis of $K D R$ mRNA expression was performed on the Roche LightCycler $^{\circledR} 480$ (Roche, Shanghai, China) using the SYBR Premix EX Taq system. The forward primer of $K D R$ was 5'-ATGCAGAGCAAGGTGCTGC-3' and the reverse primer was 5'- TTAAACAGGAGGAGAGCTCAGTG-3'. The amplification system $(20 \mu \mathrm{L})$ contained $10 \mu \mathrm{L}$ SYBR Premix EX Taq, $0.2 \mu \mathrm{L}$ of each primer $(20 \mu \mathrm{M}), 7.6 \mu \mathrm{L}$ double-distilled water, and $2 \mu \mathrm{L}$ cDNA. The mRNA expression of the $K D R$ gene was detected using the comparative $\mathrm{Ct}$ $\left(2^{-\Delta \Delta \mathrm{Ct}}\right)$. The mRNA expression of $G A P D H$ was used as an endogenous control.

\section{Statistical Analysis}

All variables in this study were statistically analyzed using the statistical analysis software SPSS version 19.0 (IBM Corp., Armonk, NY USA). The Hardy-Weinberg equilibrium test was performed for the 4397T $>C$ genotypes using the chi-squared test. The significance of observed differences in proportions was tested via the chi-squared test; Fisher's exact test was performed when data were spare. The analysis between continuous variables and 4397T $>$ C genotypes was performed using the MannWhitney U nonparametric test (between the two groups). The primary analysis was performed on PFS. The KaplanMeier curves were drawn using Stata 14.0 (Stata Corporation, College Station, TX, USA) to compare the differences in PFS and OS among patients with different genotypes. Differences in survival were compared using the Log rank test. PFS was defined as the period from the time of treatment with apatinib mesylate to disease progression or patient death due to any cause, whichever occurred first. OS was defined as the period from the time of treatment with apatinib mesylate to patient death from any cause. For those without disease progression or death by the end of the study follow-up, the survival end points were censored at the date of last follow-up. For the multivariable analysis, a Cox proportional hazards model was constructed for PFS, and the backward-selection procedure was used to adjust for potential confounding covariates. A $P<0.05$ denoted statistical significance.

\section{Results}

\section{Baseline Characteristics of I 35 Patients with NSCLC and the Genotypes of the KDR Gene 4397T>C Polymorphism}

The baseline characteristics of the 135 patients with NSCLC are shown in Table 1. The median age of the patients was 58 years (range: 22-78 years), 91 patients were males $(67.41 \%)$, and the ECOG 0 score was noted in 52 patients $(38.52 \%)$. Of the patients, 93 were nonsmokers/former smokers (68.89\%). Considering the exclusion of squamous-cell carcinoma cases, most commonly observed histology in these patients was adenocarcinoma (122 cases; 90.37\%), and large cell carcinoma (13 cases; 9.63\%). Pathological stages IIIb and IV were reported in nine and 126 cases, respectively. A positive driver gene mutation status was confirmed in 49 patients $(36.30 \%)$. Moreover, the history of previous treatment with secondand third- or subsequent-line therapy was observed in 52 
Table I Baseline Characteristics of the 135 Patients with NSCLC According to the KDR 4397T>C Polymorphism Status

\begin{tabular}{|c|c|c|c|c|}
\hline \multirow[t]{2}{*}{ Characteristics } & \multirow{2}{*}{$\begin{array}{l}\text { Total } \\
(n=\mid 35, \%)\end{array}$} & \multicolumn{2}{|c|}{ 4397T>C Genotypes } & \multirow[t]{2}{*}{$P$} \\
\hline & & TT $(n=82)$ & $\operatorname{TC} / C C(n=53)$ & \\
\hline Age, median (range) & $58(22-78)$ & $58(22-75)$ & $58(24-78)$ & 0.631 \\
\hline \multicolumn{5}{|l|}{ Gender } \\
\hline Male & $91(67.4 I)$ & $55(67.07)$ & $36(67.92)$ & 0.918 \\
\hline Female & $44(32.59)$ & $27(32.93)$ & $17(32.08)$ & \\
\hline \multicolumn{5}{|l|}{ ECOG Score } \\
\hline 0 & $52(38.52)$ & $33(40.24)$ & $19(35.85)$ & 0.608 \\
\hline $\mathrm{I}-2$ & $83(6 \mid .48)$ & $49(59.76)$ & $34(64.15)$ & \\
\hline \multicolumn{5}{|l|}{ Smoking Status } \\
\hline Nonsmoker/former smoker & $93(68.89)$ & $56(68.29)$ & $37(69.8 I)$ & 0.852 \\
\hline Smoker & $42(3 I . I I)$ & $26(31.7 I)$ & $16(30.19)$ & \\
\hline \multicolumn{5}{|l|}{ Histology } \\
\hline Adenocarcinoma & $122(90.37)$ & 75 (9l.46) & $47(88.68)$ & 0.592 \\
\hline Large cell carcinoma & $13(9.63)$ & $7(8.54)$ & $6(11.32)$ & \\
\hline \multicolumn{5}{|l|}{ Pathological Stage } \\
\hline IIlb & $9(6.67)$ & $5(6.10)$ & $4(7.55)$ & 0.742 \\
\hline IV & $126(93.33)$ & $77(93.90)$ & $49(92.45)$ & \\
\hline \multicolumn{5}{|l|}{ Driver Gene Mutation Status ${ }^{\mathrm{a}}$} \\
\hline Positive & $49(36.30)$ & 30 (36.59) & $19(35.85)$ & 0.931 \\
\hline Negative & $86(63.70)$ & $52(63.4 I)$ & $34(64.15)$ & \\
\hline \multicolumn{5}{|l|}{ History of Previous Treatment } \\
\hline Second line & $52(38.52)$ & $31(37.80)$ & $21(39.62)$ & 0.832 \\
\hline Third or subsequent line & $83(6 \mid .48)$ & $51(62.20)$ & $32(60.38)$ & \\
\hline \multicolumn{5}{|l|}{ History of Target Drug Therapy } \\
\hline Yes & $61(45.18)$ & $37(45.12)$ & $24(45.28)$ & 0.985 \\
\hline No & $74(54.82)$ & $45(54.88)$ & $29(54.72)$ & \\
\hline \multicolumn{5}{|l|}{ Initial Dosage of Apatinib Mesylate } \\
\hline $500 \mathrm{mg}$ & $73(54.07)$ & $44(53.66)$ & $29(54.72)$ & 0.904 \\
\hline $750 \mathrm{mg}$ & $62(45.93)$ & $38(46.34)$ & $24(45.28)$ & \\
\hline
\end{tabular}

Notes: ${ }^{a}$ The driver gene was EGFR, ALK, or ROSI.

Abbreviations: NSCLC, non-small cell lung cancer; KDR, kinase insert domain receptor; ECOG, Eastern Cooperative Oncology Group.

and 83 cases, respectively. A total of 61 patients had previously received targeted drug therapy (45.18\%). Apatinib mesylate was administered at an initial dosage of $500 \mathrm{mg}$ and $750 \mathrm{mg}$ in 73 and 62 cases, respectively.

Of the KDR gene polymorphisms analyzed, only 4397T $>C$ was of clinical significance. The germline mutation frequency of 4397T $>\mathrm{C}$ among the 135 patients with NSCLC was as follows: TT genotype (82 cases, $60.74 \%$ ); TC genotype (46 cases, $34.07 \%$ ); and CC genotype (seven cases, $5.19 \%$ ). Of note, the minor allele frequency of $4397 \mathrm{~T}>\mathrm{C}$ was 0.22 . The distribution of the polymorphism genotypes was in accordance with the Hardy-Weinberg equilibrium $(P=0.868)$. Considering the relatively low frequency of the $\mathrm{CC}$ genotype, the $\mathrm{TC}$ and $\mathrm{CC}$ genotypes were merged in the following analysis. As shown in Table 1, patients with the TT and TC/CC genotypes were wellbalanced with similar baseline characteristics.

\section{Influence of the KDR 4397T>C Polymorphism on the Clinical Outcomes of I 35 Patients with NSCLC}

All 135 patients included in this study were available for efficacy evaluation. Complete response (zero cases), partial response ( 25 cases), stable disease (63 cases), and progressive disease (47 cases) were evaluated according to the 
RECIST version 1.1. Therefore, the ORR was $18.52 \%$ (25/ 135 patients) and disease control rate was $65.19 \%(25+63 /$ 135 patients).

The median follow-up of all patients from the time of enrolment to the last follow-up was 24.5 months (follow-up range: 1-35 months). The median PFS (mPFS) of all 135 patients with NSCLC included in our study was 3.95 months (95\% confidence interval: 2.90-5.15). Additionally, we further analyzed the mPFS of subgroups with different baseline characteristics. As shown in Figure 2, in the univariate analysis, the mPFS of subgroups with different baseline characteristics was similar. The only exception was the ECOG score, which had a significant influence on PFS. This finding indicated that the mPFS of patients with an ECOG 0 score was longer than that of patients with ECOG $1-2$ scores ( 4.85 vs 3.35 months, respectively $P=0.011$ ).

In terms of the polymorphism analysis, as described in Figure 3, the mPFS of the patients with the TT and TC/CC genotypes of the $4397 \mathrm{~T}>\mathrm{C}$ polymorphism was 4.80 and 2.80 months, respectively; this difference was statistically significant $\left(\chi^{2}=9.50, P=0.002\right)$. Furthermore, the Cox regression model was constructed including the baseline characteristics which were significant in the univariate analysis to adjust for other confounding factors, as illustrated in Figure 2. The results of the multivariate analysis are shown in Table 2. After adjustment for other confounding factors, a statistically significant difference was observed for the influence of the 4397T $>C$ polymorphism on PFS. This finding indicated that this polymorphism was an independent factor for PFS (odds ratio=1.72, $P=0.009$ ). Additionally, after the multivariate adjustment, the ECOG score remained an independent factor for PFS (odds ratio=1.45, $P=0.015$ ).

The follow-up period of our study was sufficiently long; thus, the OS was also evaluated. The median OS (mOS) of the 135 patients with NSCLC was 10.05 months (95\% confidence interval: 8.50-10.95). Regarding the polymorphism analysis, as demonstrated in Figure 4, the mOS of the NSCLC patients with the TT and TC/CC genotypes of the $4397 \mathrm{~T}>\mathrm{C}$ polymorphism was 10.56 and 9.10 months, respectively; the difference was statistically significant $\left(\chi^{2}=3.70, P=0.041\right)$.

\section{Impact of the KDR 4397T>C Polymorphism on the Safety of Patients with NSCLC}

Furthermore, adverse reactions are an important aspect to evaluate in the clinical application of apatinib mesylate at an initial dosage of $750 \mathrm{mg}$ and $500 \mathrm{mg}$. Considering that the low incidence of adverse reactions may lead to bias in the polymorphism study, adverse events with an incidence $\geq 10 \%$ were recorded and analyzed. As shown in Table 3, there were no grade $\geq 4$ adverse events observed in our study. The most common treatment-related adverse events were hypertension $(56.30 \%)$, hand-foot syndrome $(51.11 \%)$, proteinuria (47.41\%), fatigue $(35.56 \%)$, oral mucositis $(30.37 \%)$, decreased appetite $(25.19 \%)$, diarrhea (22.96\%), hematologic toxicity (19.26\%), nausea and vomiting (14.07\%), and increased levels of transaminase (11.11\%). In terms of the 4397T $>$ C polymorphism analysis, as shown in Table 3, there was no significant difference observed in adverse events between the patients with the TT and TC/CC genotypes of the 4397T >C polymorphism.

Additionally, during treatment with apatinib mesylate, a total of 36 patients $(26.67 \%)$ were subjected to dose reduction. Of the 62 patients who initially received a dosage of $750 \mathrm{mg}, 21$ patients required dose reduction to $500 \mathrm{mg}$. Among those who received a dosage of $500 \mathrm{mg}, 15$ patients required dose reduction to $250 \mathrm{mg}$. Six patients (4.44\%) had dose discontinuity. Common causes of dose reduction or discontinuity in these patients included hypertension, proteinuria, hand-foot syndrome, and fatigue.

\section{Influence of the KDR 4397T>C Polymorphism on the mRNA Expression of the KDR Gene}

Biopsy cancer tissue specimens were collected from 66 randomly selected samples of patients with NSCLC. The mRNA expression of $K D R$ was detected using the RNA extracted from these specimens. The prevalence of the KDR 4397T $>C$ polymorphism in these 66 specimens was as follows: TT genotype (40 cases; 60.61\%), TC genotype (23 cases; $34.85 \%$ ), and CC genotype (three cases; $4.54 \%$ ). This prevalence was comparable with the genotype frequency observed among the 135 patients with NSCLC. The distribution of the three genotypes was in accordance with the Hardy-Weinberg equilibrium $(P=0.894)$. Similarly, the $\mathrm{CC}$ and TC genotypes were merged in the following analysis. As illustrated in Figure 5, in comparison to patients with the TT genotype, those with the TC/CC genotype showed higher relative expression of $K D R$ mRNA in the biopsy cancer tissue specimens (median: 4.05 ; range: $3.33-4.90$ vs median: 2.93; range: $1.95-4.15$, respectively); this difference was statistically significant $(P<0.001)$. 


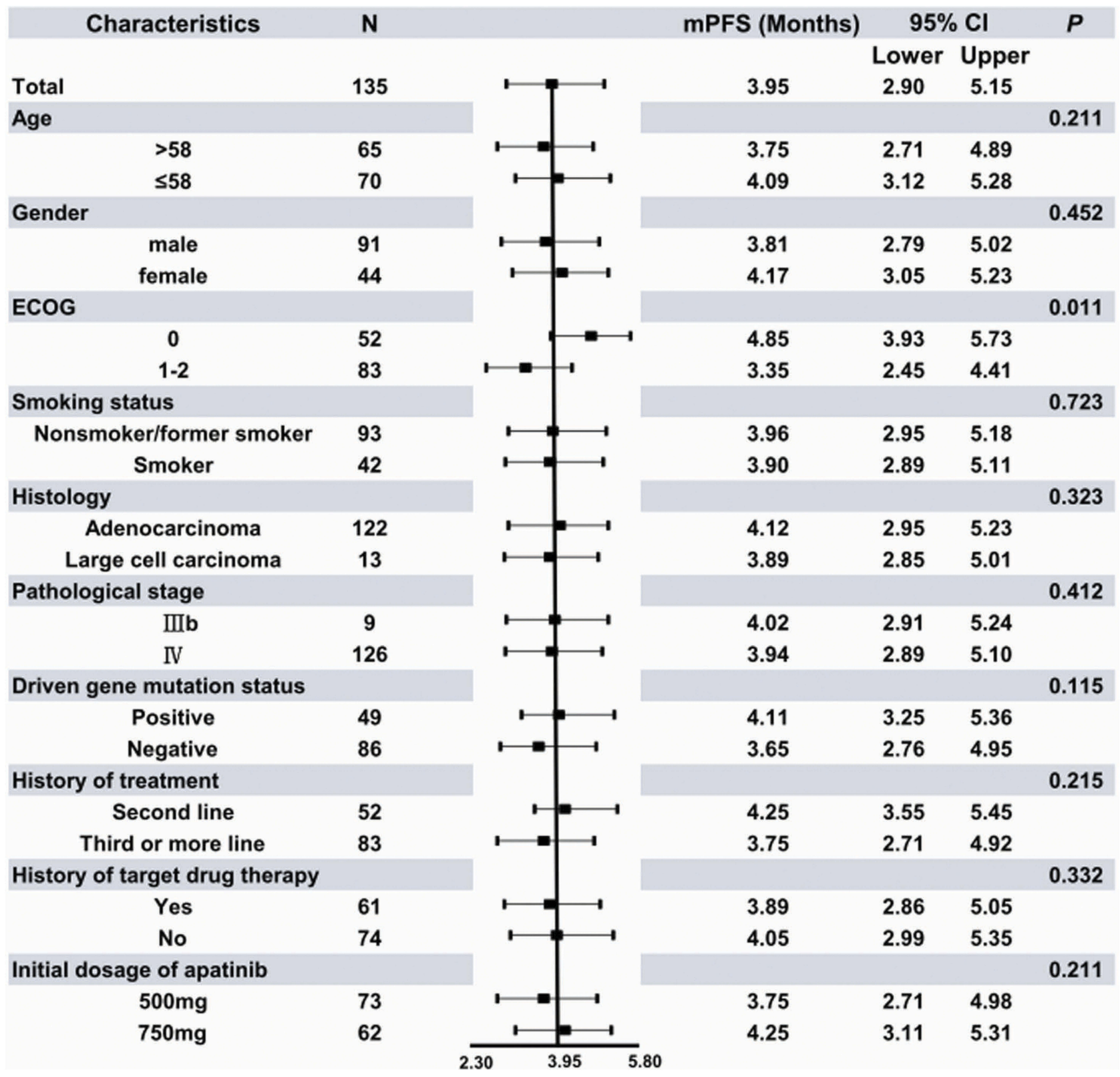

Figure 2 Forest plot of the median progression-free survival of 135 patients with NSCLC in subgroups with different baseline characteristics.

\section{Discussion}

This retrospective study provided real-world evidence regarding the clinical outcomes and safety of apatinib mesylate in the treatment of advanced NSCLC in patients who progressed after standard therapy. In the polymorphism analysis, 135 patients with NSCLC were genotyped for the KDR 4397T $>C$ polymorphism. The correlation analysis indicated that the TC/CC genotype was associated with worse PFS and OS. Furthermore, the results of the KDR mRNA expression analysis revealed that the mRNA expression levels of $K D R$ were significantly different in patients with different genotypes. The clinical outcomes of patients with advanced NSCLC who received apatinib mesylate may be influenced by the $K D R 4397 \mathrm{~T}>\mathrm{C}$ polymorphism through mediation of the $K D R$ mRNA expression.

As a small-molecule tyrosine kinase inhibitor specifically targeting $K D R$, apatinib mesylate is effective against gastric cancer, colorectal cancer, lung cancer, and soft tissue sarcoma. ${ }^{18-20}$ Since its approval by the cFDA in October 2014, a few studies investigated biomarkers of treatment with apatinib mesylate, mainly focusing on the prediction of efficacy and side effects caused by treatment 


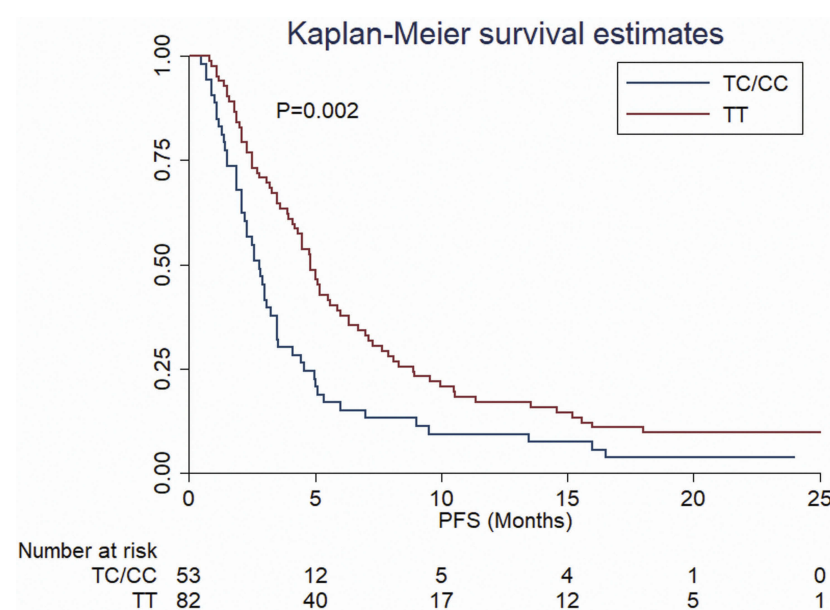

Figure 3 Progression-free survival of 135 patients with NSCLC according to the KDR 4397T >C genotype status.

with apatinib mesylate. ${ }^{21}$ Notably, the occurrence of proteinuria or hand-foot syndrome during treatment with apatinib could predict superior clinical outcomes. ${ }^{22}$ However, research focusing on the pharmacogenomics of treatment with apatinib mesylate was limited. ${ }^{23}$

Based on the clinical outcomes of patients included in this study, the ORR of 135 patients with advanced NSCLC who received treatment with apatinib mesylate was $18.52 \%$, the disease control rate was $65.19 \%$ and the mPFS was 3.95 months, which was slightly lower than that reported in the first Phase II clinical trial of apatinib mesylate in advanced NSCLC initiated by Zhang et al in 2012 . $^{24}$ Owing to the retrospective design of the study, we hypothesized that patient management was not sufficient and normative compared with the phase II clinical trial, as shown in the other retrospective study. ${ }^{25}$ Unlike in the phase II clinical trial conducted by Zhang et al, patients with an ECOG 2 score were also included in our study. The influence of the ECOG score on patient prognosis has

Table 2 Multivariate Cox Regression Analysis of PFS According to the Baseline Characteristics and 4397T>C Polymorphism

\begin{tabular}{|l|l|l|l|}
\hline Characteristics & OR $(\mathbf{9 5 \%}$ Cl) & df & P \\
\hline $\begin{array}{c}\text { ECOG } \\
0\end{array}$ & $\begin{array}{l}\text { I (reference) } \\
\text { I-45 (I.25-I.I.7I) }\end{array}$ & I & 0.015 \\
\hline $\begin{array}{l}\text { 4397T>C Genotype } \\
\text { TT genotype } \\
\text { TC/CC genotype }\end{array}$ & $\begin{array}{l}\text { I (reference) } \\
\text { I.72 (I.4I-I.97) }\end{array}$ & I & 0.009 \\
\hline
\end{tabular}

Abbreviations: ECOG, Eastern Cooperative Oncology Group; OR, odds ratio; $\mathrm{Cl}$, confidence interval; $d f$, degree of freedom.

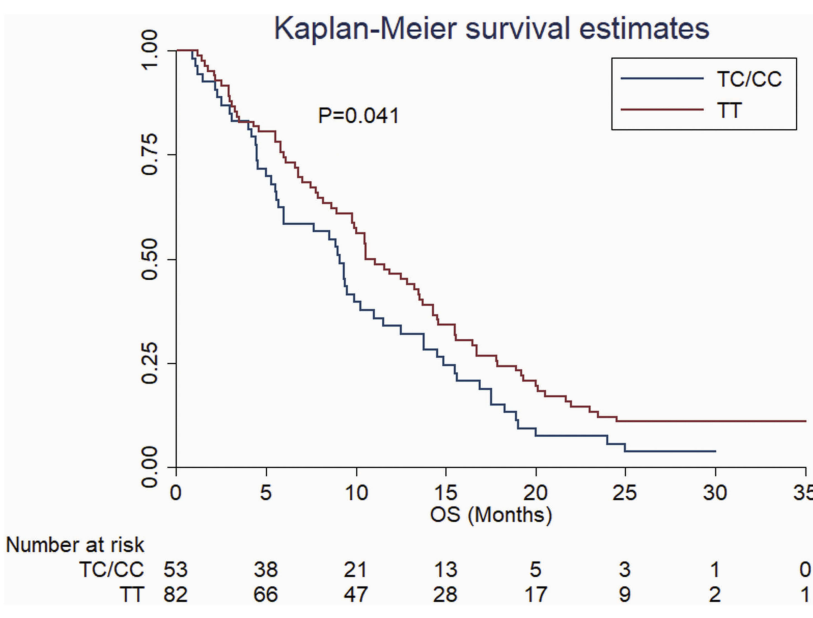

Figure 4 Overall survival of 135 patients with NSCLC according to the KDR 4397T >C genotype status.

been confirmed in numerous studies; high scores have been associated with worse prognosis. ${ }^{26}$ However, the clinical outcomes of other retrospective analyses of apatinib mesylate in the treatment of NSCLC were consistent with the results of the present study. ${ }^{27,28}$ Interestingly, the mOS in our study was 10.05 months, which was slightly longer than that reported in other research studies of apatinib mesylate. ${ }^{29,30}$ We speculate that this effect may be attributed to the continued approval of immunotherapy drugs and anlotinib in China in 2018 Consequently, patients with NSCLC had the opportunity to receive immunotherapy and other targeted drugs in subsequentline treatment, which had been shown to be effective and offered survival benefit to the patients. ${ }^{31}$

Furthermore, the results of the polymorphism analysis in our study were partly consistent with those of a previous study conducted by Scartozzi et al. ${ }^{32}$ A total of 148 patients with advanced hepatocellular cancer who received sorafenib were included in the ALICE-1 study. The results showed that the prevalence of the rs 2071559 polymorphism in the study population was 0.49 , which was markedly higher than the minor allele frequency $(0.22)$ recorded in our study. Moreover, the results of that study also showed that the prognosis of patients with the TC/CC genotype was worse, which was consistent with the findings of our study. However, the previous study failed to identify the mechanisms of influence of the polymorphism on the prognosis of patients with hepatocellular carcinoma who received treatment with sorafenib. Moreover, the results of a recent study conducted by Sullivan et al also supported our findings. ${ }^{33}$ A total of 170 patients with metastatic NSCLC treated with first-line platinum-based chemotherapy were included in 
Table 3 Correlation Analysis Between the KDR 4397T>C Polymorphism and the Adverse Events with an Incidence $\geq 10 \%$

\begin{tabular}{|c|c|c|c|c|c|c|}
\hline \multirow[t]{2}{*}{ Adverse Events } & \multirow[t]{2}{*}{ Grade I-2 } & \multirow[t]{2}{*}{ Grade 3} & \multirow[t]{2}{*}{ Total (n, \%) } & \multicolumn{2}{|c|}{ 4397T>C Genotypes } & \multirow[t]{2}{*}{$P$} \\
\hline & & & & TT $(n=82)$ & $\operatorname{TC} / C C(n=53)$ & \\
\hline Hypertension & 58 (42.97) & $18(13.33)$ & $76(56.30)$ & $46(56.10)$ & $30(56.60)$ & 0.954 \\
\hline Hand-foot syndrome & $52(38.52)$ & $17(12.59)$ & $69(5 \mathrm{I} .1 \mathrm{I})$ & $45(54.88)$ & $24(45.28)$ & 0.276 \\
\hline Proteinuria & $54(40.00)$ & $10(7.4 I)$ & $64(47.4 I)$ & 40 (48.78) & $24(45.28)$ & 0.691 \\
\hline Fatigue & $42(31.11)$ & $6(4.44)$ & $48(35.56)$ & $30(36.59)$ & $18(33.96)$ & 0.756 \\
\hline Oral mucositis & 41 (30.37) & $0(0.00)$ & $4 \mathrm{I}(30.37)$ & $26(3 I .7 I)$ & $15(28.30)$ & 0.674 \\
\hline Decreased appetite & $32(23.7 I)$ & $2(1.48)$ & $34(25.19)$ & $22(26.83)$ & $12(22.64)$ & 0.584 \\
\hline Diarrhea & $28(20.74)$ & $3(2.22)$ & 31 (22.96) & $19(23.17)$ & $12(22.64)$ & 0.943 \\
\hline Hematologic toxicity & $22(16.30)$ & $4(2.96)$ & $26(19.26)$ & $17(20.73)$ & $9(16.98)$ & 0.589 \\
\hline Nausea and vomiting & $19(14.07)$ & $0(0.00)$ & $19(14.07)$ & $12(14.63)$ & $7(|3.2|)$ & 0.816 \\
\hline Increased levels of transaminase & I5 (II.II) & $0(0.00)$ & $15(I I . I I)$ & $10(12.20)$ & $5(9.43)$ & 0.618 \\
\hline
\end{tabular}

Abbreviation: $K D R$, kinase insert domain receptor.

that study, and the polymorphisms of vascular endothelial growth factor A (VEGFA), VEGFR1, and VEGFR2 were genotyped. The results of the multivariate analysis demonstrated that VEGFA rs2010963 and VEGFR2 rs2071559 were significantly associated with OS. Specifically, patients

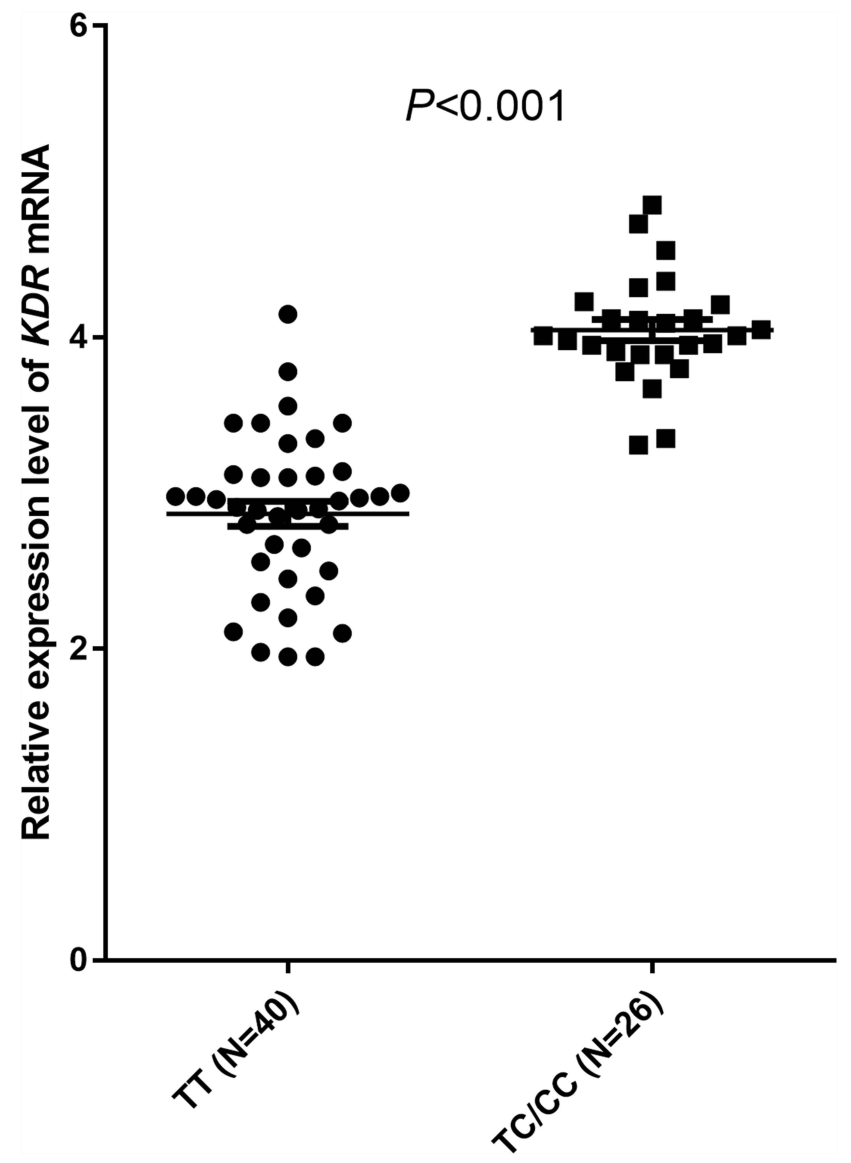

Figure 5 Relative expression levels of KDR mRNA in 66 biopsy cancer tissue specimens according to the KDR 4397T >C polymorphism status. with the rs2071559 major genotype were associated with better prognosis. This was consistent with our results indicating that patients with the TT genotype show superior clinical outcomes. Another recent Phase III trial conducted by George et $\mathrm{al}^{34}$ investigated adjuvant sunitinib in patients with high-risk renal cell carcinoma. The results showed that longer disease-free survival was observed with sunitinib versus placebo in patients with the VEGFR2 rs2071559 TT genotype. Moreover, the stratification analysis based on the rs2071559 genotype showed that the median diseasefree survival was longer in patients with the TT genotype than in the total-study population after treatment with sunitinib. This finding was in accordance with the results of our study. Based on the above evidence, the KDR 4397T $>\mathrm{C}$ polymorphism may be an independent biomarker for the prediction of patients with NSCLC who received apatinib mesylate as subsequent-line therapy.

In terms of the safety analysis, a previous study found that hypertension was usually the most common adverse reaction in other tumors following treatment with apatinib mesylate. ${ }^{35}$ Consistently, our study indicated that hypertension was the most commonly noted adverse event. Similar to another previous study of apatinib mesylate, other adverse events with an incidence $\geq 10 \%$ were hand-foot syndrome, proteinuria, fatigue, oral mucositis, decreased appetite, and diarrhea. ${ }^{36}$ The correlation analysis between polymorphism and adverse events failed to show statistical significance, indicating that the polymorphism was not associated with drug disposition.

Additionally, a total of 66 randomly selected specimens of biopsy cancer tissue obtained from patients with NSCLC were also included in our mRNA expression 
analysis. The results indicated that the mRNA expression of the $K D R$ gene was significantly higher in patients carrying the $\mathrm{C}$ allele. $K D R$ is the receptor with the strongest binding ability for $V E G F-A$ and plays the most important role in signal transmission. Moreover, its expression levels play a crucial role in the process of angiogenesis. ${ }^{37}$ It has been shown that high levels of $K D R$ expression in tumor cells facilitate the regeneration of blood vessels, increasing the risk of relapse and metastasis. $^{38,39}$ Some research studies showed that high expression of the KDR gene was associated with worse PFS and OS in patients with $\mathrm{NSCLC}^{40}$ and cervical cancer. $^{41}$ These findings are consistent, to some extent, with those of our study.

Inevitably, this study was characterized by some limitations. Firstly, the sample size of this study was small. A study including a larger sample size is required to evaluate the efficacy of apatinib mesylate in patients with NSCLC and the prognostic significance of the polymorphism. Additionally, our study was designed as a retrospective analysis; thus, some bias may have been unavoidable. However, the prognostic significance of the polymorphism was fully evaluated, and the KDR gene mRNA expression was determined to reveal the role of the polymorphism in prognosis.

\section{Conclusion}

The results of our study are clinically significant for the evaluation of prognosis in patients with NSCLC receiving apatinib mesylate. This evidence may assist clinicians in identifying sensitive patients who could benefit from treatment with apatinib mesylate. The clinical outcomes of treatment with apatinib mesylate for advanced non-squamous NSCLC in patients who progressed after standard therapy may be influenced by the $K D R 4397 \mathrm{~T}>\mathrm{C}$ polymorphism through mediation of the mRNA expression of $K D R$.

\section{Acknowledgment}

This study was supported by a grant from Cancer Research Program of National Cancer Center (NCC2017A30).

\section{Disclosure}

The authors report no conflicts of interest in this work.

\section{References}

1. Bray F, Ferlay J, Soerjomataram I, et al. Global cancer statistics 2018: GLOBOCAN estimates of incidence and mortality worldwide for 36 cancers in 185 countries. CA Cancer J Clin. 2018;68:394-424. doi: $10.3322 /$ caac.v68.6
2. Chen W, Zheng R, Baade PD, et al. Cancer statistics in China, 2015. CA Cancer J Clin. 2016;66:115-132. doi:10.3322/caac.21338

3. Remon J, Ahn MJ, Girard N, et al. Advanced-stage non-small cell lung cancer: advances in thoracic oncology 2018. J Thorac Oncol. 2019;14:1134-1155. doi:10.1016/j.jtho.2019.03.022

4. Martinez P, Peters S, Stammers T, et al. Immunotherapy for the first-line treatment of patients with metastatic non-small cell lung cancer. Clin Cancer Res. 2019;25:2691-2698. doi:10.1158/10780432.CCR-18-3904

5. Sandler A, Gray R, Perry MC, et al. Paclitaxel-carboplatin alone or with bevacizumab for non-small-cell lung cancer. $N$ Engl $J$ Med. 2006;355:2542-2550. doi:10.1056/NEJMoa061884

6. Zhou C, Wu YL, Chen G, et al. Beyond: a randomized, double-blind, placebo-controlled, multicenter, phase III study of first-line carboplatin/paclitaxel plus bevacizumab or placebo in chinese patients with advanced or recurrent nonsquamous non-small-cell lung cancer. J Clin Oncol. 2015;33:2197-2204. doi:10.1200/JCO.2014.59.4424

7. Han B, Li K, Wang Q, et al. Effect of anlotinib as a third-line or further treatment on overall survival of patients with advanced non-small cell lung cancer: the ALTER 0303 Phase 3 randomized clinical trial. JAMA Oncol. 2018;4:1569-1575. doi:10.1001/ jamaoncol.2018.3039

8. Li J, Qin S, Xu J, et al. Randomized, double-blind, placebo-controlled phase III trial of apatinib in patients with chemotherapy-refractory advanced or metastatic adenocarcinoma of the stomach or gastroesophageal junction. $J$ Clin Oncol. 2016;34:1448-1454. doi:10.1200/JCO.2015.63.5995

9. Wen S, Shao G, Zheng J, et al. Apatinib regulates the cell proliferation and apoptosis of liver cancer by regulation of VEGFR2/STAT3 signaling. Pathol Res Pract. 2019;215:816-821. doi:10.1016/j. prp.2019.01.021

10. Zhao D, Hou H, Zhang X. Progress in the treatment of solid tumors with apatinib: a systematic review. Onco Targets Ther. 2018;11:4137-4147. doi:10.2147/OTT

11. Villaruz LC, Socinski MA. The role of anti-angiogenesis in non-small-cell lung cancer: an update. Curr Oncol Rep. 2015;17:26. doi:10.1007/s11912-015-0448-y

12. Liu X, Qin S, Wang Z, et al. Correction to: early presence of anti-angiogenesis-related adverse events as a potential biomarker of antitumor efficacy in metastatic gastric cancer patients treated with apatinib: a cohort study. J Hematol Oncol. 2018;11:5. doi:10.1186/ s13045-017-0545-5

13. Liu S, Wu M, Zhang B, et al. Analysis of genetic polymorphisms for age-related macular degeneration (AMD) in Chinese Tujia ethnic minority group. BMC Med Genet. 2019;20:25. doi:10.1186/s12881019-0756-4

14. Babyshkina N, Zavyalova M, Tarabanovskaya N, et al. Predictive value of vascular endothelial growth factor receptor type 2 in triple-negative breast cancer patients treated with neoadjuvant chemotherapy. Mol Cell Biochem. 2018;444:197-206. doi:10.1007/ s11010-017-3244-1

15. Escudier B, Rini BI, Motzer RJ, et al. Genotype correlations with blood pressure and efficacy from a randomized phase III trial of second-line axitinib versus sorafenib in metastatic renal cell carcinoma. Clin Genitourin Cancer. 2015;13:328-337.e323. doi:10.1016/j.clgc.2015.02.007

16. Eisenhauer EA, Therasse P, Bogaerts J, et al. New response evaluation criteria in solid tumours: revised RECIST guideline (version 1.1). Eur J Cancer. 2009;45:228-247. doi:10.1016/j.ejca.2008.10.026

17. Miller TP, Fisher BT, Getz KD. et al. Unintended consequences of evolution of the common terminology criteria for adverse events. Pediatr Blood Cancer. 2019; e27747. doi:10.1002/pbc.27747.

18. Tian Z, Gu Z, Wang X, et al. Efficacy and safety of apatinib in treatment of osteosarcoma after failed standard multimodal therapy: an observational study. Medicine (Baltimore). 2019;98:e15650. doi:10.1097/MD.0000000000015650 
19. Chen X, Qiu T, Zhu Y, et al. A single-arm, phase II study of apatinib in refractory metastatic colorectal cancer. Oncologist. 2019.

20. Liao Z, Li F, Zhang C, et al. Phase II trial of VEGFR2 inhibitor apatinib for metastatic sarcoma: focus on efficacy and safety. Exp Mol Med. 2019;51:24. doi:10.1038/s12276-019-0221-7

21. Chen LT, Oh DY, Ryu MH, et al. Anti-angiogenic therapy in patients with advanced gastric and gastroesophageal junction cancer: a systematic review. Cancer Res Treat. 2017;49:851-868. doi:10.4 $143 /$ crt.2016.176

22. Liu X, Qin S, Wang Z, et al. Early presence of anti-angiogenesis-related adverse events as a potential biomarker of antitumor efficacy in metastatic gastric cancer patients treated with apatinib: a cohort study. J Hematol Oncol. 2017;10:153. doi:10.1186/s13045-017-0521-0

23. Fang SC, Huang W, Zhang YM, et al. Hypertension as a predictive biomarker in patients with advanced non-small-cell lung cancer treated with apatinib. Onco Targets Ther. 2019;12:985-992. doi:10.2147/ OTT.S189984

24. Ma JT, Sun J, Sun L, et al. Efficacy and safety of apatinib in patients with advanced nonsmall cell lung cancer that failed prior chemotherapy or EGFR-TKIs: a pooled analysis. Medicine (Baltimore). 2018;97:e12083. doi:10.1097/MD.0000000000012083

25. Wei JH, Feng ZH, Cao Y, et al. Predictive value of single-nucleotide polymorphism signature for recurrence in localised renal cell carcinoma: a retrospective analysis and multicentre validation study. Lancet Oncol. 2019;20:591-600. doi:10.1016/S1470-2045(18) 30932-X

26. Agemi Y, Shimokawa T, Sasaki J, et al. Prospective evaluation of the G8 screening tool for prognostication of survival in elderly patients with lung cancer: a single-institution study. PLoS One. 2019;14: e0210499. doi:10.1371/journal.pone.0210499

27. Zhang D, Zhang C, Huang J, et al. Clinical investigation of the efficacy and toxicity of apatinib (YN968D1) in stage III/IV non-small cell lung cancer after second-line chemotherapy treatment: a retrospective study. Thorac Cancer. 2018;9:1754-1762. doi:10. 1111/tca.2018.9.issue-12

28. Liu Z, Ou W, Li N, et al. Apatinib monotherapy for advanced non-small cell lung cancer after the failure of chemotherapy or other targeted therapy. Thorac Cancer. 2018;9:1285-1290. doi:10.1111/ tca.2018.9.issue- 10

29. Wu F, Zhang S, Xiong A, et al. A phase II clinical trial of apatinib in pretreated advanced non-squamous non-small-cell lung cancer. Clin Lung Cancer. 2018;19:e831-e842. doi:10.1016/j.cllc.2018.06.002

30. Wu D, Liang L, Nie L, et al. Efficacy, safety and predictive indicators of apatinib after multilines treatment in advanced nonsquamous nonsmall cell lung cancer: apatinib treatment in nonsquamous NSCLC. Asia Pac J Clin Oncol. 2018;14:446-452. doi:10.1111/ajco.12870
31. Nadal E, Massuti B, Domine M, et al. Immunotherapy with checkpoint inhibitors in non-small cell lung cancer: insights from long-term survivors. Cancer Immunol Immunother. 2019;68:341-352. doi:10.1007/s00262-019-02310-2

32. Scartozzi M, Faloppi L, Svegliati Baroni G, et al. VEGF and VEGFR genotyping in the prediction of clinical outcome for HCC patients receiving sorafenib: the ALICE-1 study. Int $J$ Cancer. 2014;135:1247-1256. doi:10.1002/ijc.v135.5

33. Sullivan I, Riera P, Andres M, et al. Prognostic effect of VEGF gene variants in metastatic non-small-cell lung cancer patients. Angiogenesis. 2019;22:433-440. doi:10.1007/s10456-019-09668-y

34. George DJ, Martini JF, Staehler M, et al. Phase III trial of adjuvant sunitinib in patients with high-risk renal cell carcinoma: exploratory pharmacogenomic analysis. Clin Cancer Res. 2019;25:1165-1173. doi:10.1158/1078-0432.CCR-18-1724

35. Zhu Y, Feng B, Mei L, et al. Clinical efficacy of TACE combined with Apatinib in the treatment of advanced hepatocellular carcinoma. J BUON. 2019;24:608-614.

36. Xie L, Xu J, Sun X, et al. Apatinib for advanced osteosarcoma after failure of standard multimodal therapy: an open label phase II clinical trial. Oncologist. 2018;24(7):e542-50.

37. Cho HD, Moon KD, Park KH, et al. Effects of auriculasin on vascular endothelial growth factor (VEGF)-induced angiogenesis via regulation of VEGF receptor 2 signaling pathways in vitro and in vivo. Food Chem Toxicol. 2018;121:612-621. doi:10.1016/j. fct.2018.09.025

38. Lu Y, Xu Q, Zuo Y, et al. Isoprenaline/beta2-AR activates Plexin-A1/ VEGFR2 signals via VEGF secretion in gastric cancer cells to promote tumor angiogenesis. BMC Cancer. 2017;17:875. doi:10.1186/ s12885-017-3894-0

39. Jinesh GG, Manyam GC, Mmeje CO, et al. Surface PD-L1, E-cadherin, CD24, and VEGFR2 as markers of epithelial cancer stem cells associated with rapid tumorigenesis. Sci Rep. 2017;7:9602. doi:10.1038/s41598-017-08796-z

40. Ding M, Liu L, Hu C, et al. Expression of VEGFR2 and NRP-1 in non-small cell lung cancer and their clinical significance. Chin J Cancer Res. 2014;26:669-677. doi:10.3978/j.issn.1000-9604.2014.12.04

41. Dang YZ, Zhang Y, Li JP, et al. High VEGFR1/2 expression levels are predictors of poor survival in patients with cervical cancer. Medicine (Baltimore). 2017;96:e5772. doi:10.1097/MD.000000 0000005772
OncoTargets and Therapy

\section{Publish your work in this journal}

OncoTargets and Therapy is an international, peer-reviewed, open access journal focusing on the pathological basis of all cancers, potential targets for therapy and treatment protocols employed to improve the management of cancer patients. The journal also focuses on the impact of management programs and new therapeutic agents and protocols on patient perspectives such as quality of life, adherence and satisfaction. The manuscript management system is completely online and includes a very quick and fair peer-review system, which is all easy to use. Visit http://www.dovepress.com/ testimonials.php to read real quotes from published authors. 